\title{
PARTITION PROPERTIES ON COUNTABLE BIPARTITE GRAPHS
}

\author{
BORIS ŠOBOT \\ Received 19 March, 2014
}

\begin{abstract}
We classify all countably infinite (on both sides) bipartite graphs $G$ having a naturally defined version of the pigeonhole principle.

2010 Mathematics Subject Classification: 05C63; 03E02

Keywords: bipartite graphs, partition properties
\end{abstract}

\section{INTRODUCTION}

Definition 1. An $\left(\boldsymbol{\aleph}_{0}, \boldsymbol{\aleph}_{0}\right)$-bigraph is a structure $G=(X, Y, E)$, where $(X \cup Y, E)$ is a digraph such that $E \subseteq\{x y: x \in X, y \in Y\}$ and $|X|=|Y|=\aleph_{0}$. We call $(X, Y)$ the bipartition of $G, X$ the left side, and $Y$ the right side.

The complement $\bar{G}$ of a bigraph $G=(X, Y, E)$ has the same set of vertices with the same bipartition, and $(X \times Y) \backslash E$ for the set of edges.

We say that a countable structure $S$ has property $\mathcal{P}$ (also known as the pigeonhole principle) if for every partition of its domain into finitely many pieces at least one of the induced substructures is isomorphic to $S$. This property was investigated for various graph-theoretic structures. We list some such results.

Theorem 1 ([2], Proposition 3.4). The only countable graphs with the property $\mathcal{P}$ up to isomorphism are the empty graph, the complete graph and the Rado graph.

Theorem 2 ([1], Theorem 1). The only countable tournaments with the property $\mathcal{P}$ up to isomorphism are the random tournament and tournaments $\omega^{\alpha}$ and $\left(\omega^{\alpha}\right)^{*}$ for $0<\alpha<\omega_{1}$.

Theorem 3 ([3], Corollary 2.4). The only countable digraphs with the property $\mathcal{P}$ up to isomorphism are the empty digraph, the random tournament, tournaments $\omega^{\alpha}$ and $\left(\omega^{\alpha}\right)^{*}$ for $0<\alpha<\omega_{1}$, the random digraph, the random acyclic digraph and its inverse.

Research supported by the MNTRRS (Project 174006: Set Theory, Model Theory and Set-theoretic Topology). 
As we shall see, for bigraphs it makes sense only to consider certain altered property that we will call $\mathcal{P}^{\prime}$. It is our goal to classify all countable bigraphs with property $\mathcal{P}^{\prime}$.

Since random bigraphs will play an important role in our classification, we will now list some of their properties. More on these structures can be found in [5].

Definition 2. A $\left(\boldsymbol{\aleph}_{0}, \boldsymbol{\aleph}_{0}\right)$-bigraph $(X, Y, E)$ is $\left(\boldsymbol{\aleph}_{0}, \boldsymbol{\aleph}_{0}\right)$-random if

$\forall U, W \in[Y]^{<\aleph_{0}}(U \cap W=\varnothing \Rightarrow \exists x \in X(\forall u \in U x u \in E \wedge \forall w \in W x w \notin E))$.

$(X, Y, E)$ is $\left(\boldsymbol{\aleph}_{0}, \boldsymbol{\aleph}_{0}\right)$-dense if

$\forall U, W \in[X]^{<\aleph_{0}}(U \cap W=\varnothing \Rightarrow \exists y \in Y(\forall u \in U u y \in E \wedge \forall w \in W w y \notin E))$.

If $G$ satisfies both these conditions we will call it $\left(\boldsymbol{\aleph}_{0}, \boldsymbol{\aleph}_{0}\right)$-random dense.

Proposition 1. Let $G=(X, Y, E)$ be a $\left(\boldsymbol{\aleph}_{0}, \boldsymbol{\aleph}_{0}\right)$-random bigraph. Then every $y \in Y$ is of infinite degree in both $G$ and $\bar{G}$.

Clearly, the same results hold for vertices in $X$ if $G$ is $(\kappa, \lambda, \mu)$-dense. In [4] it was shown that there is exactly one $\left(\boldsymbol{\aleph}_{0}, \boldsymbol{\aleph}_{0}\right)$-random dense bigraph up to isomorphism. This is not true for $\left(\boldsymbol{\aleph}_{0}, \boldsymbol{\aleph}_{0}\right)$-random bigraphs.

\section{THE CLASSIFICATION}

Now we turn to our main concern, a partition property resembling $\mathcal{P}$.

Definition 3. Let $G_{1}=\left(X_{1}, Y_{1}, E_{1}\right)$ and $G_{2}=\left(X_{2}, Y_{2}, E_{2}\right)$ be bigraphs. A bijection $f: X_{1} \cup Y_{1} \rightarrow X_{2} \cup Y_{2}$ is an isomorphism if for all $x \in X_{1}, y \in Y_{1}: x y \in E_{1}$ iff $f(x) f(y) \in E_{2}$.

Of course, for $\left(\boldsymbol{\aleph}_{0}, \boldsymbol{\aleph}_{0}\right)$-bigraphs it makes sense only to consider partitions into $\left(\boldsymbol{\aleph}_{0}, \boldsymbol{\aleph}_{0}\right)$-bigraphs (otherwise we could split the bigraph in two parts, each having one finite side). So we say that an $\left(\boldsymbol{\aleph}_{0}, \boldsymbol{\aleph}_{0}\right)$-bigraph $G$ has property $\mathcal{P}^{\prime}$ if:

$\mathcal{P}^{\prime}$ : for every partition of the set of vertices of $G$ into finitely many pieces that each induce $\left(\boldsymbol{\aleph}_{0}, \boldsymbol{\aleph}_{0}\right)$-bigraphs at least one of the induced sub-bigraphs is isomorphic to $G$.

Example 1. $S=(X, Y, E)$ is defined by $X=\left\{x_{n}: n \in \omega\right\}, Y=\left\{y_{n}: n \in \omega\right\}$ and $E=\left\{x_{n} y_{0}: n \in \omega\right\} . S$ has the property $\mathcal{P}^{\prime}$.

It is easy to see that if $G$ has $\mathcal{P}$, then its complement $\bar{G}$ and the bigraph $G^{*}$ obtained by reversing all the edges also have $\mathcal{P}^{\prime}$.

Taking into account Propositions 1, 2 and 3 it would be natural to expect the $\left(\boldsymbol{\aleph}_{0}, \boldsymbol{\aleph}_{0}\right)$-random dense bigraph has the property $\mathcal{P}^{\prime}$. However, this is not the case. 
Lemma 1. The $\left(\boldsymbol{\aleph}_{0}, \aleph_{0}\right)$-random dense bigraph does not satisfy $\mathcal{P}^{\prime}$.

Proof. Let $y \in Y$ be arbitrary and let $X_{1}$ be the set of all its neighbors. By Proposition 1, both $X_{1}$ and $X \backslash X_{1}$ are infinite. Let $x \in X_{1}$ and let $Y_{0}$ be the set of all its neighbors. If we partition vertices of $G$ into $\left(X \backslash X_{1}\right) \cup Y_{0}$ and $X_{1} \cup\left(Y \backslash Y_{0}\right)$, in both induced sub-bigraphs we have an isolated vertex, so none of those parts can be isomorphic to $G$.

In order to prove our main result, Theorem 4, we will consider several cases concerning the minimal degree of a vertex in $G$, and the number of vertices of minimal degree. $d_{G}(v)$ will denote the degree of a vertex $v$ in $G$, and we will drop the $G$ if it is clear from the context.

Lemma 2. If all the vertices in both $G$ and $\bar{G}$ are of infinite degree, then $G$ does not satisfy $\mathcal{P}^{\prime}$.

Proof. Let $G=(X, Y, E)$ be an $\left(\aleph_{0}, \aleph_{0}\right)$-bigraph with property $\mathcal{P}^{\prime}$ such that all the vertices in both $G$ and $\bar{G}$ are of infinite degree. We prove that $G$ must be $\left(\boldsymbol{\aleph}_{0}, \boldsymbol{\aleph}_{0}\right)$ random, and in a similar way it is proved that it must be $\left(\boldsymbol{\aleph}_{0}, \boldsymbol{\aleph}_{0}\right)$-dense. This will be a contradiction with Lemma 1.

Let $u_{1}, u_{2}, \ldots, u_{m}, w_{1}, w_{2}, \ldots, w_{n} \in Y$. Suppose there is no $x \in X$ connected to all $u_{1}, u_{2} \ldots, u_{m}$, and not connected to any of $w_{1}, w_{2} \ldots, w_{n}$. We define the partition $X=X_{1} \cup \cdots \cup X_{m} \cup X_{1}^{\prime} \cup \cdots \cup X_{n}^{\prime}$ as follows. First we enumerate the vertices in $X$ : $X=\left\{x_{k}: k \in \omega\right\}$, and begin with $X_{1}, \ldots, X_{m}, X_{1}^{\prime}, \ldots, X_{n}^{\prime}$ empty. We proceed by recursion on $k$. If there is $i \in\{1,2, \ldots, m\}$ such that $x_{k} u_{i} \notin E$, let $r_{k}$ be such $i$ that $X_{i}$ is of the smallest cardinality (if there are several such $i$, choose any of them). On the other hand, if there is $j \in\{1,2, \ldots, n\}$ such that $x_{k} w_{j} \in E$, let $s_{k}$ be such $j$ that $X_{j}^{\prime}$ is of the smallest cardinality. Now, if there is no $j$ as above, put $x_{k}$ in the set $X_{r_{k}}$; if there is no $i$ as above then put $x_{k}$ in the set $X_{s_{k}}^{\prime}$. Otherwise, if $\left|X_{r_{k}}\right| \leq\left|X_{s_{k}}^{\prime}\right|$, put $x_{k}$ in the set $X_{r_{k}}$, and otherwise in the set $X_{s_{k}}^{\prime}$.

What we get in the end are disjoint sets $X_{1}, \ldots, X_{m}, X_{1}^{\prime}, \ldots, X_{n}^{\prime}$, each $X_{i}$ containing no neighbors of $u_{i}$ and each $X_{i}^{\prime}$ containing no non-neighbors of $w_{i}$. In addition, each $X_{i}$ is infinite; suppose not and let, for example, $X_{i}$ be the one with the smallest cardinality (the case when there are several such sets is considered similarly). Let $k_{0} \in \omega$ be big enough such that by the $k_{0}$-th step of the recursion all the elements of $X_{i}$ and at least $\left|X_{i}\right|+1$ elements of other sets were added to them. Then for $k>k_{0}$ we would have no more elements $x_{k}$ such that $x_{k} u_{i} \notin E$, which is impossible because $u_{i}$ has infinitely many non-neighbors.

Now we partition $Y$ into infinite pieces: $Y=Y_{1} \cup \cdots \cup Y_{m} \cup Y_{1}^{\prime} \cup \cdots \cup Y_{n}^{\prime}$ arbitrarily so that $u_{i} \in Y_{i}$ and $w_{i} \in Y_{i}^{\prime}$. Finally, we split $G$ into $m+n$ parts induced by $X_{i} \cup Y_{i}$ and $X_{i}^{\prime} \cup Y_{i}^{\prime}$. Clearly, none of the obtained sub-bigraphs can be isomorphic to $G$, since each of them either has an isolated vertex or a vertex connected to all the vertices from the opposite side, a contradiction. 
Let $G=(X, Y, E)$ be an $\left(\boldsymbol{\aleph}_{0}, \boldsymbol{\aleph}_{0}\right)$-bigraph. We denote $D_{X}=\{d(v): v \in X\}$, $D_{Y}=\{d(v): v \in Y\}, d_{X}=\min D_{X}$ and $d_{Y}=\min D_{Y}$.

Lemma 3. If in an $\left(\boldsymbol{\aleph}_{0}, \boldsymbol{\aleph}_{0}\right)$-bigraph $G=(X, Y, E)$ that satisfies $\mathcal{P}^{\prime}$ holds $d_{X} \in$ $\omega \backslash\{0\}$ or $d_{Y} \in \omega \backslash\{0\}$, then $G$ is isomorphic either to $S$ or to $S^{*}$.

Proof. Suppose $d_{X} \in \omega \backslash\{0\}$. We will consider the following cases:

Case $1^{\circ}$ There is exactly one vertex in $X$ of degree $d_{X}$, say $x$. Split $G$ arbitrarily into sub-bigraphs $G_{1}$ and $G_{2}$. Let, for example, $x \in G_{1}$.

If $G_{1}$ is isomorphic to $G$, we move one of the neighbors of $x$ (call it $y$ ) to $G_{2}$. If now $G_{2}+y$ is isomorphic to $G$, it has a vertex $x_{2}$ of degree $d_{X}$, so we also move one of the neighbors of $x_{2}$ from $G_{1}$ to $G_{2}$.

If, on the other hand, $G_{2}$ is isomorphic to $G$ then there is $x_{2} \in X$ that has degree $d_{X}$ in $G_{2}$. Now by moving one of the neighbors of $x_{2}$ (say $y$ ) from $G_{1}$ to $G_{2}$ we get a partition in which $G_{2}$ has no vertices of degree $d_{X}$. If now the other part $\left(G_{1}-y\right)$ is isomorphic to $G$, we move one of the neighbors of $x$ in the same direction.

Either way, we arrive at a partition into two parts, none of which is isomorphic to $G$.

Case $2^{\circ}$ There are at least two (but finitely many) vertices in $X$ of degree $d_{X}$, say $x_{1}, x_{2}, \ldots, x_{k}$. Split $G$ into two $\left(\aleph_{0}, \aleph_{0}\right)$-bigraphs $G_{1}$ and $G_{2}$ arbitrarily, but in such way that in each of the parts there is at least one of the vertices $x_{1}, x_{2}, \ldots, x_{k}$. Let $G_{1}$ be isomorphic to $G$; then there are exactly $k$ vertices of degree $d_{X}$ on its left side. Let there be $l$ vertices of degree $d_{X}$ on the left side of $G_{2}$. If $l \geq k$ move one of the vertices $x_{1}, x_{2}, \ldots, x_{k}$ from $G_{1}$ to $G_{2}$; otherwise move one of them in the other direction. In the partition obtained in this way none of the parts can be isomorphic to $G$.

Case $3^{\circ}$ There are infinitely many vertices in $X$ of degree $d_{X}$. If $d_{X}>1$ then it is easy to split $G$ so that there are two vertices in different parts such that each of them has in the induced sub-bigraph degree less than $d_{X}$. Now let $d_{X}=1$. If there is no $y \in Y$ connected to all of the vertices of degree 1, we can split so that in both parts we get isolated vertices on the left side. So let $y \in Y$ be connected to all vertices of degree 1 in $X$. There may be no other vertices in $X$, because otherwise we could split into two parts $G_{1}$ and $G_{2}$ so that in $G_{1}$ we get isolated vertices and in $G_{2}$ having on the right side more that one vertex connected to vertices of degree 1 . It follows that all the other vertices on the right side must be isolated, so the only remaining bigraph is $S$.

The corresponding cases when $d_{Y} \in \omega \backslash\{0\}$ are considered analogously and we get the bigraph $S^{*}$ as another solution.

This also takes care of the corresponding cases for $\bar{G}$ (i.e. when on one side of $\bar{G}$ there are vertices of finite degree, but no isolated vertices) and gives us two more solutions: $\bar{S}$ and $\bar{S}^{*}$. For the discussion in the upcoming theorem we will also need 
a property stronger than $\mathcal{P}^{\prime}$ :

$\mathcal{P}^{\prime \prime}$ : for every partition of the set of vertices of $G$ into finitely many pieces that each induce $\left(\boldsymbol{\aleph}_{0}, \boldsymbol{\aleph}_{0}\right)$-bigraphs all of the induced sub-bigraphs are isomorphic to $G$.

It is easy to see that, when investigating either of the properties $\mathcal{P}^{\prime}$ and $\mathcal{P}^{\prime \prime}$, the condition is not weakened when "finitely many pieces" is replaced with "two pieces".

Lemma 4. The only $\left(\boldsymbol{\aleph}_{0}, \boldsymbol{\aleph}_{0}\right)$-bigraphs with the property $\mathcal{P}^{\prime \prime}$ up to isomorphism are the empty $\left(\boldsymbol{\aleph}_{0}, \boldsymbol{\aleph}_{0}\right)$-bigraph and the complete $\left(\boldsymbol{\aleph}_{0}, \boldsymbol{\aleph}_{0}\right)$-bigraph.

Proof. The cases $d_{X} \in \omega \backslash\{0\}$ and $d_{Y} \in \omega \backslash\{0\}$ are discussed in Lemma 3 and yield no solutions ( $S$ and $S^{*}$ clearly do not satisfy $\mathcal{P}^{\prime \prime}$ ).

Let $d_{X}=0$. The case when there are finitely many isolated vertices is easy: partition in an arbitrary way, and if both parts are isomorphic to $G$, then move one of the vertices that were isolated in $G$ from one part to the other. If infinitely many vertices in $X$ are isolated, we can split so that one of the parts is an empty bigraph, so $G$ itself must be empty.

If in $\bar{G}$ there are vertices of finite degree, the proof is analogous, and gives us the complete bigraph. Finally, if both $G$ and $\bar{G}$ have only vertices of infinite degree, by Lemma 2 we have no further solutions.

Theorem 4. The only $\left(\boldsymbol{\aleph}_{0}, \boldsymbol{\aleph}_{0}\right)$-bigraphs with the property $\mathcal{P}^{\prime}$ up to isomorphism are the empty $\left(\boldsymbol{\aleph}_{0}, \boldsymbol{\aleph}_{0}\right)$-bigraph, the complete $\left(\boldsymbol{\aleph}_{0}, \boldsymbol{\aleph}_{0}\right)$-bigraph, the bigraphs $S$ and $S^{*}$ and their complements.

Proof. Let $G=(X, Y, E)$ be an $\left(\aleph_{0}, \aleph_{0}\right)$-bigraph with property $\mathcal{P}^{\prime}$. Since some of the cases were analyzed in Lemmas 2 and 3, we consider cases when one of the $d_{X}$ and $d_{Y}$ is 0 , and the other is not in $\omega \backslash\{0\}$.

Case $1^{\circ}$ Either $X$ or $Y$ has exactly one isolated vertex, say $x \in X$. We claim that $G-x$ (the bigraph obtained by removing $x$ from $G$ ) has the stronger property $\mathcal{P}^{\prime \prime}$. To prove it, suppose $G-x$ is partitioned into two parts, thus inducing sub-bigraphs $G_{1}$ and $G_{2}$. If we add $x$ as an isolated vertex to $G_{1}$, we get a partition of $G$ into $G_{1}+x$ and $G_{2}$. One of the parts must be isomorphic to $G$. Suppose it is $G_{2}$. Then, if we split $G$ into $G_{1}$ and $G_{2}+x, G_{1}$ must also be isomorphic to $G$. Hence each of the bigraphs $G_{1}$ and $G_{2}$ has exactly one isolated vertex, call them $x_{1}$ and $x_{2}$. But now, if we split $G$ into $G_{1}-x_{1}$ and $G_{2}+x+x_{1}$, none of the two induced parts is isomorphic to $G$, a contradiction. So $G_{1}+x$ must be isomorphic to $G$, and it follows that $G_{1}$ is isomorphic to $G-x$. In an analogous way we prove that $G_{2}$ is also isomorphic to $G-x$.

By Lemma $4 G-x$ must be either empty or complete. The first possibility would mean that there were more than one isolated vertices in $X$, a contradiction. So $G$ is isomorphic to $\bar{S}^{*}$. 
If, instead of $X$, the right side $Y$ has exactly one isolated vertex, we get $\bar{S}$ as a solution.

Case $2^{\circ}$ There are at least two (but finitely many) isolated vertices in either $X$ or $Y$; this is analogous to Case $2^{\circ}$ of Lemma 3.

Case $3^{\circ}$ There are infinitely many isolated vertices in both $X$ and $Y$. Then we can split $G$ into two empty sub-bigraphs. None of the parts is isomorphic to $G$, except if $G$ itself is empty.

Case $4^{\circ}$ There are infinitely many isolated vertices in $X$, and $Y$ contains only vertices of infinite degree (or the other way around). Let $\left\langle x_{k}: k<\omega\right\rangle$ be an enumeration of the vertices from the left side. Again we will construct, by recursion on $k$, a partition that will be a counterexample for $\mathcal{P}^{\prime}$. To begin with, let the sets $X_{0}, X_{1}, Y_{0}$ and $Y_{1}$ be empty. If $x_{k}$ has "unused" neighbors from $Y$ (not yet placed into $Y_{0}$ or $Y_{1}$ ), put $x_{k}$ into $X_{1}$ and put one of its neighbors into $Y_{1}$; otherwise just place $x_{k}$ in $X_{0}$. When we're finished, we add the rest of the vertices from $Y$ to $Y_{0}$. Hence we obtained a partition of $G$ into two parts, one of them containing no edges and the other without isolated vertices, which is a contradiction.

The corresponding cases for $\bar{G}$ (when $\bar{G}$ has isolated vertices) are discussed as above, so we also get the complete $\left(\aleph_{0}, \aleph_{0}\right)$-bigraph.

\section{ACKNOWLEDGMENT}

The author wishes to thank the referee for his valuable suggestions.

\section{REFERENCES}

[1] A. Bonato, P. Cameron, and D. Delić, "Tournaments and orders with the pigeonhole property." Canad. Math. Bull., vol. 43, pp. 397-405, 2000, doi: 10.4153/CMB-2000-047-6.

[2] P. Cameron, "The random graph," in The Mathematics of Paul Erdos, Vol. 2, R. L. Graham and J. Nešetřil, Eds. Berlin: Springer, 1997, ch. 7, pp. 333-351, doi: 10.1007/978-1-4614-7254-4.

[3] R. Diestel, I. Leader, A. Scott, and S. Thomassé, "Partitions and orientations of the Rado graph." Trans. Am. Math. Soc., vol. 359, no. 5, pp. 2395-2405, 2007, doi: 10.1090/S0002-9947-06-040864.

[4] M. Goldstern, R. Grossberg, and M. Kojman, "Infinite homogeneous bipartite graphs with unequal sides." Discrete Math., vol. 149, no. 1-3, pp. 69-82, 1996, doi: 10.1016/0012-365X(94)00339-K.

[5] B. Šobot, "Random bipartite graphs." Novi Sad J. Math., vol. 44, no. 2, pp. 69-75, 2014.

Author's address

Boris Šobot

University of Novi Sad, Department of Mathematics and Informatics, Trg Dositeja Obradovića 4, 21000 Novi Sad, Serbia

E-mail address: sobotedmi.uns.ac.rs 